\title{
.Parametric Modelling as a Design Representation in Architecture: A Process Account
}

\begin{tabular}{|c|c|c|}
\hline Robert Woodbury & Shane Williamson & Philip Beesley \\
Canadian Design Research & Faculty of Architecture & School of Architecture \\
Network & Landscape and Design & University of Waterloo \\
Simon Fraser University & University of Toronto & 7 Melville Street South \\
Central City & 230 College Street & Cambridge ON N1S 2H4 \\
250-13450 102nd Avenue & Toronto ON M5T 1R2 & pbeesley@uwaterloo.ca \\
SurreyBC V3T OA3 & shane.williamson@utoronto.ca & \\
rw@sfu.ca & & \\
& & \\
& & \\
\end{tabular}

\begin{abstract}
Disciplines outside of engineering, for instance, architecture, are adopting parametric modelling as a design representation. This paper reports on three aspects of the adoption process, which is largely being conducted through multi-day workshops outside of formal university course settings. Fist, the structure and process of such workshops may be a model for interdisciplinary learning and university-industry liaison. Second, students learning parametric modelling must master skill different from that required for non-parametric representation. Third, the parametric modelling strategies being developed in architecture may have both similarities and differences to those used in engineering.
\end{abstract}

\section{Introduction}

Every designer both uses and develops representations. Even within drawing conventions, designers edit both work and ways in which drawing conveys the work. By editing a design we transform it to a new design. By editing a representation we change what the representation can convey about the design. We refine, restructure and re-conceive, all through the act of editing what we have made before. When we use digital media, we edit by changing the objects the medium supports and by creating views of objects that convey particular messages. We might move lines, shape surfaces, carve solids and change perspective or projection. In most systems these are isolated acts - we must separately edit all the parts of a work. Parametric modelling systems allow designers to model classes of design and parts of the editing process. Using them, designers design both their work and how it can change in its context.

At its core, parametric modelling introduces a single new idea. Relate the parts together and defer to the system the task of keeping parts so related. One idea. One change to current system design. A small change in the way we work. It turns out that this single change has far reaching effects on the way designers work, on what designers need to know, on what can be expressed and built, and on the systems needed to represent designs. Some of the very earliest design systems were parametric and the defense, marine and automotive industries have built parametric models for many years. In architecture and other areas in which designs are particular to context, the advantages of parametric modelling were long overwhelmed by complexity and cost. Our design systems persisted in providing object-by-object manual editing. In 2006 we are in the midst of rapid change in design, its means of realization and its supporting media. A small number of firms lead by setting new norms of richness and complexity in form. Graduate students from a few institutions are exploring new forms and new system ideas. Computer numerical control machinery enables fabrication of complex designs direct from computer representation. The cost of such fabrication is dropping dramatically and thus opening new realms of practical possibility. By supporting designers in explicitly stating intentions, parametric modelling systems engender exploration of new and rich formal possibilities. 


\section{Parametric Modelling}

From a user's perspective there are two types of parametric modelling systems: propagation-based and constraint-based systems. Propagation systems compute from knowns to unknowns with a dataflow model, whereas constraint systems solve sets of continuous and discrete constraints. For simplicity, albeit with some loss of generality, the following discussion is limited to propagation-based systems.

A spreadsheet is a parametric model. It maintains relationships between, say, a column of numbers and the sum of the column. The spreadsheet user builds relationships by entering formulae into cells. The user sees cells organized into tables; the computer arranges cells into networks. In both spreadsheets and designbased parametric modelling systems, work is divided between user and computer with the user defining the cell contents and network structure and the computer maintaining the values in the network. We say that data flows through the network and call both the diagram and the computational process a dataflow. Figure 1 demonstrates the shared structure of both a spreadsheet and a parametric model.

\begin{tabular}{|l|l|}
\hline & $\mathrm{A}$ \\
\hline 1 & 1 \\
\hline 2 & 1 \\
\hline 3 & $=\mathrm{A} 1+\mathrm{A} 2$ \\
\hline 4 & $=\mathrm{A} 2+\mathrm{A} 3$ \\
\hline 5 & $=\mathrm{A} 3+\mathrm{A} 4$ \\
\hline & $=$ sum(A1:A5) \\
\hline
\end{tabular}

(a) User-entered formulae in spreadsheet cells.

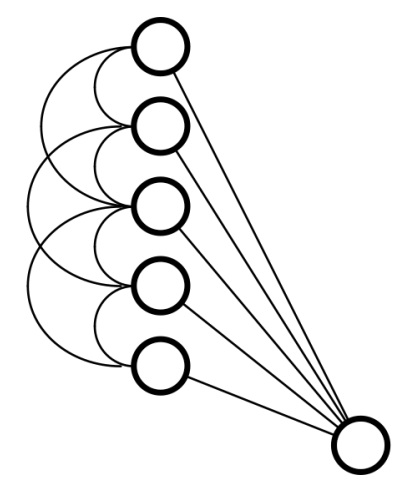

(c) The internal data structure. Circles are cells. Lines and arcs are dataflows. In this diagram, arrows are omitted: data always moves downward.

\section{Figure 1: The structure of parametric modelling.}

The key element in a parametric modelling system is thus the cell, the device by which users form relationships. In Figure 2 below, cell $\boldsymbol{b}$ receives data from cells $\boldsymbol{a}_{1}$ to $\boldsymbol{a}_{n}$. The designer using a parametric modelling system must describe both the cells from which data is received and how the data is used to compute a value for $\boldsymbol{b}$.

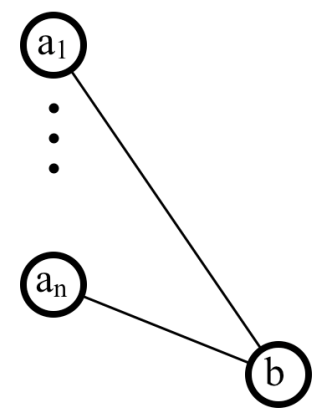
Figure 2: Cell $b$ requires an algorithm for
computing its values from its inputs.

That's it. That is all that parametric modelling is, all that it provides for the designer and all that it does. Yet, in practice these systems are complex; they require considerable effort to learn and working with them is very different from working with other systems. Why? The answer is in the cell: there is a vast range of geometric ideas that can be modeled and a number of different ways that cell formulae can be written. In addition, parametric modelling systems almost universally provide designers with the ability to devise their own computations to put inside cells. With this capability, a cell could theoretically compute, say, an entire structure for a building.

Parametric models imply families of designs. By varying the inputs to a model, different specific designs are produced. Exploring the resulting design space is one of the grand challenges for future parametric modelling research.

The engineering disciplines have long used parametric modelling software, and it may come as a surprise to engineering researchers that this class of systems has been largely absent in architecture and building design. Recently, this has been changing and several classes of systems have emerged. Systems such as Revit [1] combine data structures representing building elements (so-called Building Information Modelling (BIM)) with two-way constraints. CATIA [2] imposes a strong abstract structuring of data with access to a constraint solver and sophisticated graphical debugging methods. 
SolidWorks [3] has become nearly standard in mechanical engineering, but is largely absent in architecture. Generative Components [4] is a new system, as of Summer 2006 in beta-test, from Bentley Systems Inc. It combines a propagation-based parametric modelling system, a set of geometric types and a layered structure of access to the system through programming languages of increasing sophistication.

In May 2006, the Canadian Design Research Network hosted a Parametric Design Workshop in conjunction with the Subtle Technology Symposium ( http://www.subtletechnologies.com ). The workshop was attended by graduate students and design practitioners. It is of interest to CDEN in three ways. First, it is a model for intense interaction between design students (in our case mostly graduate students) and design practices seeking to engage with new technology. As such, it is serves to establish and strengthen productive relations between academia and practice. Second, parametric modelling systems require that students learn a new complex of skill and knowledge. The needed combination of 3D visualization, linear algebra, calculus, parametric representation and programming presents challenging issues for course design in the area. Third, architectural designers use parametric modelling strategies that are analogous to classical engeineering techniques. We demonstrate workshop outcomes in all of these aspects.

\section{The Workshops as Learning Events}

Both the SmartGeometry and CDRN workshops were held over several days in a dedicated space. For most participants the location was a retreat from daily work and family responsibilities, which enabled work with minimal distractions. SmartGeometry was held over four days in a conference centre in Cambridge England. The CDRN workshop took place over three days at the Faculty of Architecture Landscape and Design at the University of Toronto. The events were catered, the SmartGeometry event entirely so, the CDRN event for lunch and dinner only. Expectations were expressed that participants were expected to start early and finish late (for some people this translated to 18-hour days). In both workshops participation was by application. SmartGeometry as an established event received over 150 international applications for 65 positions. The CDRN workshop was organized on a very short time frame and accepted all 35 qualified applicants (all were Canadian), though this took it above its planned enrolment limit of 30. Participants included graduate students, faculty, practitioners and, in the case of the CDRN workshop, several artists and filmmakers. All participants brought their own laptops to the event. Facilitating the workshop were experienced users of parametric modelling who acted as tutors. Smart Geometry had 21 tutors for 65 students; CDRN had five (5) tutors for 35 students. These two ratios represent the extremes at the many similar workshops that have been held in recent years.

The main task of the workshop was to achieve a self-defined design goal. Some explicit instruction occurred as a plenary group during the early part of the workshop, with short demonstrations being given throughout. Small group seminars on particular topics were held as needed. Most instructional effort went into one-to-one sessions with students on their specific design problems. Especially at the SmartGeometry event there were daily reviews of progress, with a final review demonstrating all student work. An online repository of work with a very simple submission process enabled students to post work-in-progress at regular intervals. This repository (at http://smartgeometry.designscience.ca ) forms the record of work done. Not all students availed themselves of the repository. The SmartGeometry record is more complete as a full-time communications coordinator had responsibility for encouraging participants to post their work, both during and after the event.

The multi-day format, the venue as retreat, the catered meals, the long hours, the low participant/tutor ratios, the focus on individual projects and the group reviews of work all contributed to encourage an intense learning experience with considerable interaction amongst participants.

\section{Learning Parametric Modelling}

Effective use of a parametric modeler requires a practical understanding of such concepts as a vector, the cross-product, projection, parametric functions and Frenet frames. As anyone who has studied linear algebra knows, these concepts require some sophistication to master, that is, to use effectively and with control. In addition, software engineering concepts such as encapsulation and modularity are critical to making large, well-controlled parametric models. Most designers have not had much formal education in mathematics, computing or software engineering. Anecdotally we note that most workshop participants are able to gain these concepts in practice, perhaps without being able even to name them, but being able to predict their important geometric effects. It seems clear to us that it may well be appropriate to structure an introduction to these key concepts, at least for designers, around the act of parametric modelling. 
Though the first author at least has had extensive experience with such teaching, this point remains a conjecture whose confirmation (or not) will need careful instructional and quasi-experimental design.

\section{Form-finding}

Like any design representation, parametric modelling can be used towards several ends. From the current series of development workshops, it appears that, after basic learning, a motivation for many is form-finding, the use of a hypothesized external influence to guide the physical shape and material composition of a design. Form-finding is an extremely common strategy in engineering design, where the word optimization labels a specific formal device for discovery of utility-maximizing solutions to a set of constraints. In architecture, some of he best uses of the strategy have occurred in collaboration with engineering structural designers (cite Frei Otto Olympic Stadium, Foster's British Museum - visual form-finding). Arguably the best examples are from building types in which many of the structural and architectural problems are solved within the same technical system. In architecture at least, the design problems are seldom so clear. A typical situation is one of multiple design goals (for instance, identity, accommodation, privacy, access, lighting, ventilation and structure) are met in multiple technical systems (for instance, massing (overall form), spatial organization, structure, cladding, internal partitions, mechanical systems, lighting systems). Further, many goals are only discovered in the process of design. The architectural task is to make a whole, composed of many separate parts, that performs acceptably against many distinct, and potentially vague, goals.

Another possible explanation for the scarcity of form-finding in architectural process is the modelling required to state a design problem as a form-finding or optimization problem. First, there must be a precisely stated problem (goals and technical systems). Second, there must be a model, a mathematical representation of both goals and systems. Third, there must be an algorithm capable of generating model solutions.

Yet there is motivation for form-finding. Understanding implications from goals to form is historically one of the most difficult aspects of the architectural design process. The reasons are simple: design criteria are highly contextual and adequate analysis tools have been scarce. Any ability to reason for a hypothesized influence to a designed form is welcomed by at least a part of the design community.;
The criteria in form-finding may not be purely technical. The British Museum roof (Figure 3) provides a dramatic example. Its configuration was determined by a relaxation algorithm, in which the goal criterion was visual continuity, not structure. Structural strength was gained partly by sectional properties - some of the corner members are nearly solid steel.

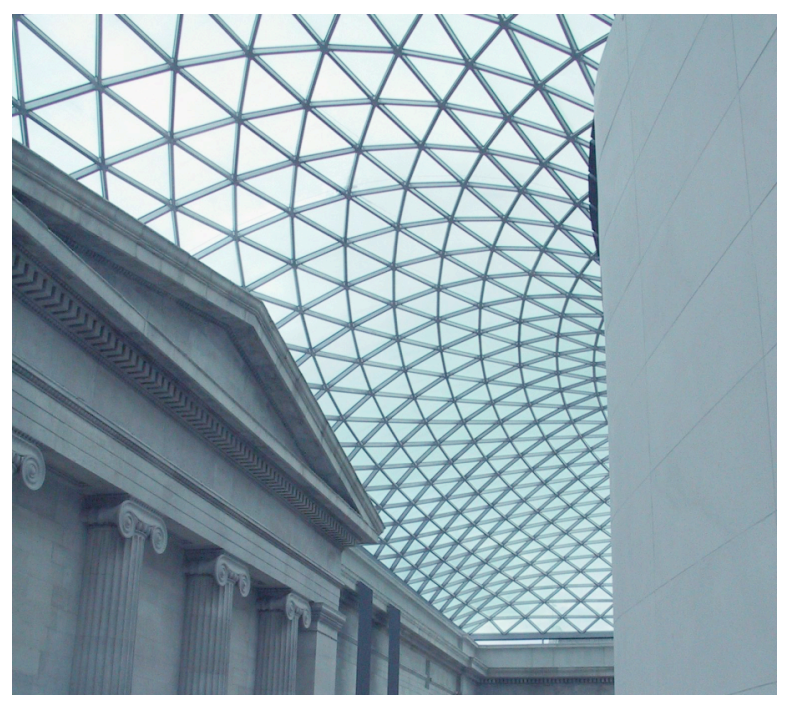

Figure 3: The roof over the courtyard of the British Museum (photo by R. Woodbury).

Parametric modelling appears to lower barriers to entry of form-finding strategies in architectural design. By making it possible to model an external effect and a design's response, parametric modelling allows access to a space of adaptive designs that would be very difficult to reach with current conventional computer-based design representations.

To support this claim, we present data from two recent parametric design workshops in which practitioners and graduate students gathered for several days to learn parametric modelling. Workshop format - differences between SmartGeometry and CDRN. Both workshops were provided with a webbased repository for recording work in progress and final results. Participants could place work in versions at any time in this repository and were encouraged to do so by the workshop facilitators. Participation in the repository was voluntary. The work in the repository was classified into categories of learning, modelling, composition, form-finding, tool making and unable to judge. The learning category describes work whose apparent intent is simply to learn about parametric modelling. Work in the modelling category aimed at replicating an existing idea or form. Composition refers to representing a class of related designs, typically using a rule or procedure for generating 
members of the class. Form-finding describes work in which an external influence shapes a design. The toolbuilding category labels work focused on developing algorithms for parametric modelling. Three works in the SmartGeometry workshop were coded as both tool-building and another category (either formfinding or modelling). Table 1 outlines the number of participants in each workshop coded into each category.

\begin{tabular}{|l|c|c|}
\hline & CDRN & SmartGeometry \\
\hline Participants & 35 & 68 \\
\hline $\begin{array}{l}\text { Submitted } \\
\text { work }\end{array}$ & 22 & 55 \\
\hline Learning & 6 & 5 \\
\hline Modelling & 2 & 12 \\
\hline Composition & 7 & 16 \\
\hline Form-finding & 6 & 13 \\
\hline Tool-building & 0 & 9 \\
\hline $\begin{array}{l}\text { Unable to } \\
\text { judge }\end{array}$ & 1 & 3 \\
\hline
\end{tabular}

Table 1: Categorization of work from the CDRN and SmartGeometry Workshops

The CDRN workshop had 35 participants., of which 22 contributed work to the repository. Of these six (6) demonstrated form-finding as the primary strategy in their work. In comparison the SmartGeometry workshop had 68 participants, of which 55 contributed work. Of these 12 demonstrated form-finding as the primary strategy.

\section{Examples of form-finding}

In this section we present three examples of formfinding from the two workshops. Dr Wassim Jabi from NJIT worked on the configuration of sun-shading devices to be applied across curved surface facades. The aim was less the design of a sun-shade with specific but more exploration of three-dimensional architectural possibilities and of modelling and programming possibilities engendered by parametric modelling. In Dr Jabi's words (used with permission):

The idea behind the exercise was twofold: 1) to create a controller (Figure 4) that would accurately supply azimuth and altitude angles for a location of a light vector (the sun). The controller consisted of two circles (one horizontal and one vertical) along which two points traveled. A third point $(S)$ on a virtual concentric sphere derived its location from the two control points.
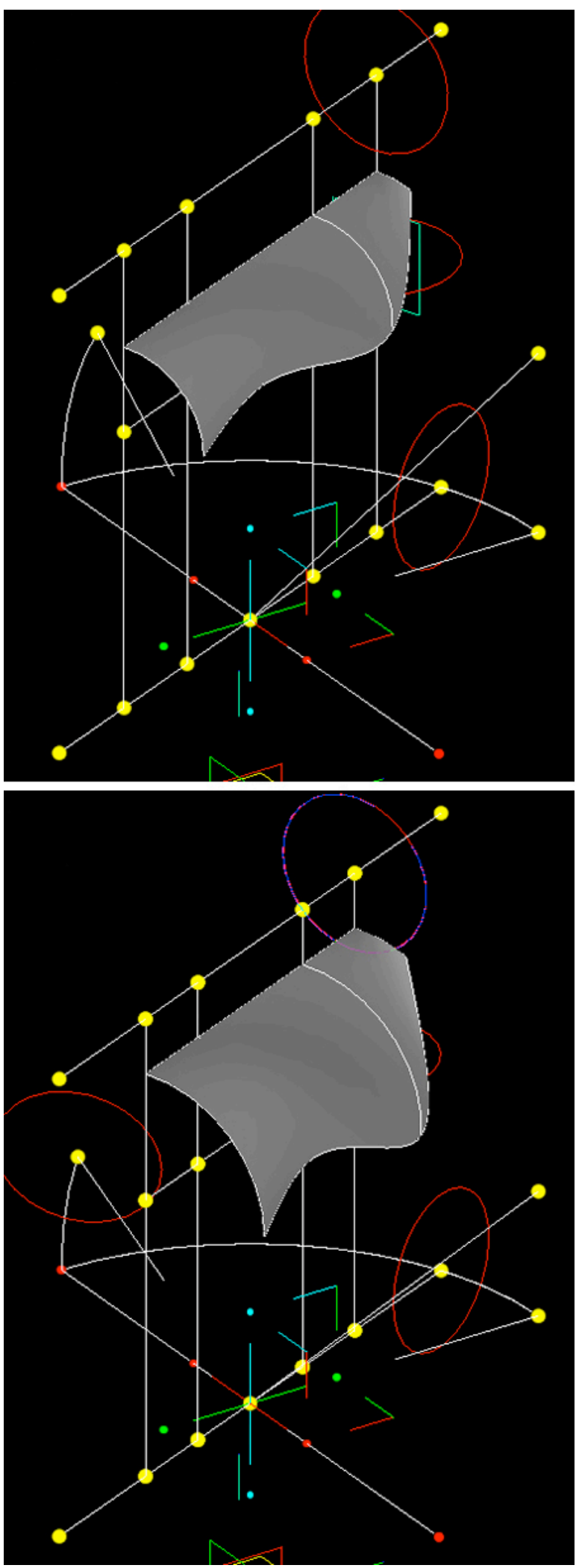

Figure 4: A parametric sunshade design (Wassim Jabi) (with permission of author). 
The sun direction was interpreted to be the direction of a line from the point $(S)$ to the center of the model. The controller and the resulting direction were used to control a shading device (Figure 5). The shading device was designed as a surface controlled by two shapes: 1) a rectangle that represented a window on a wall, and 2) a $3^{\text {rd }}$ order Bezier curve in front of the rectangle. The surface of the shading device is the result of an interpolation between the rectangle and the Bezier curve. The distance of the control poles of the Bezier curve from the wall surface behind them was controlled by the direction of the sun. More specifically, the lower points were controlled by the azimuth while the top points were controlled by the altitude of the sun. Effectively, the shading device acted as a sail that would attempt to protect the surface behind it from the sun. As the sun rose in the sky (larger altitude) the shading device would effectively "fold" back and vice versa. Each side of the shading device operated independently and extended depending on the azimuth (horizontal) angle of the sun (Figure 6).

The last step of the exercise remains largely unresolved due to time. It is to populate an undulating surface with this shading device and, based on the location of the shading device on the surface (and thus the sun incident angle), the shading device would modify its shape accordingly (Figure 7).

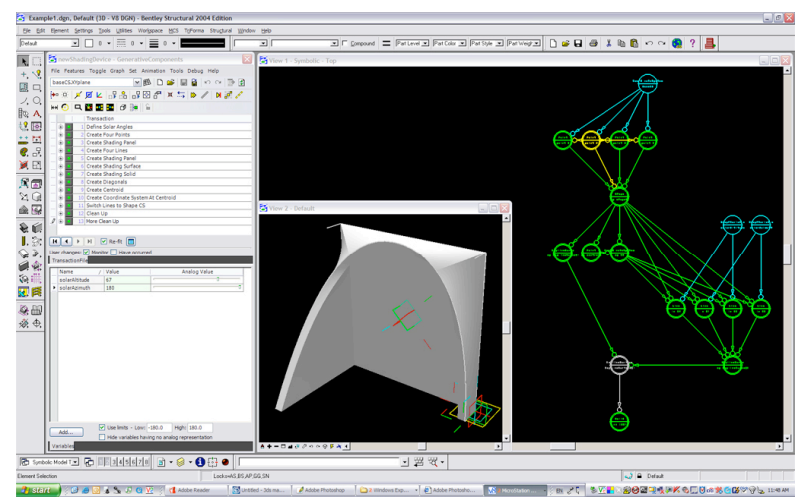

Figure 5: A refined sunshade and its parametric model. (Wassim Jabi) (with permission of author).
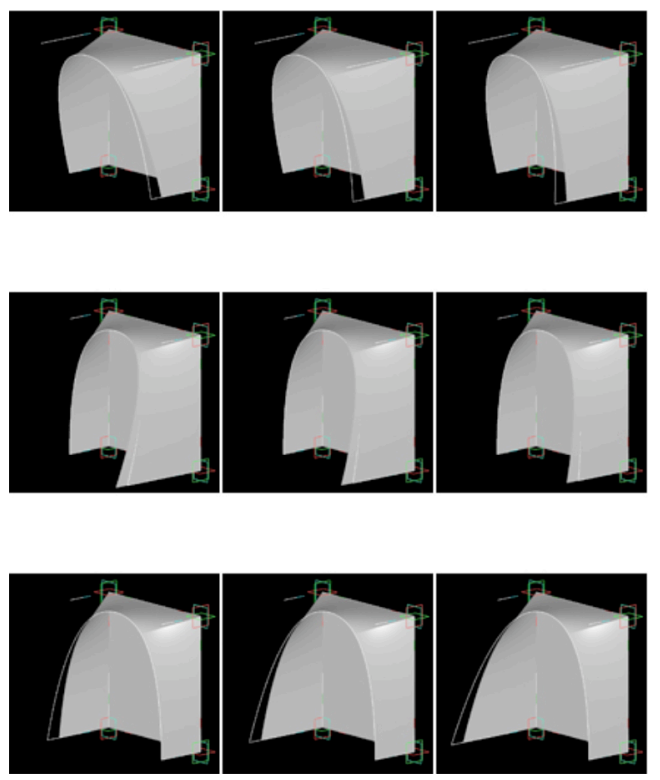

Figure 6: Alternative sunshade designs (Wassim Jabi) (with permission of author).

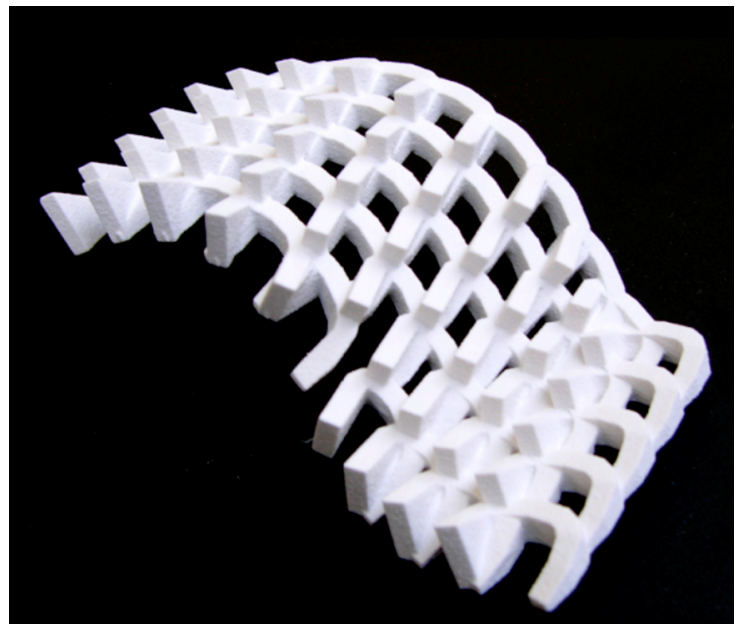

Figure 7: Sunshades arrayed across a curved surface. The photograph is of a 3D print made on rapid prototyping machine. (Wassim Jabi) (with permission of author).

Another example, also from SmartGeometry, is that of Matthew Jogan, in which the external influence is viewing quality in a theatre house and the driven design is the form of the theatre seating (Figure 8). In his words

The form of a theater is a direct result of the relationship between the performance space and viewing locations in a theater house. My project is part of a continuing study to use parametric modeling to derive theater form structures from generative sight line criteria. It builds upon previous parametric work that embedded 
generative seating layouts with ticket pricing structures to study the relation between various seating layouts and their resulting affect on house revenue. The workshop project uses Generative Components to study methods for analyzing and promoting individual view locations to their appropriate relative view position, given a proposed row configuration and view range. One method involves height promotion through the analysis of an individual view location and its potential view obstructions within a proposed seating configuration. The second method creates view positions by generating seating tier structures from a sectional relation of view positions. In addition to these studies, subcomponents were created that can determine and analyze view constrains between a view location and a given view range. This resulting data can then be used for selection set criteria and for visual analysis of a generative theater form.
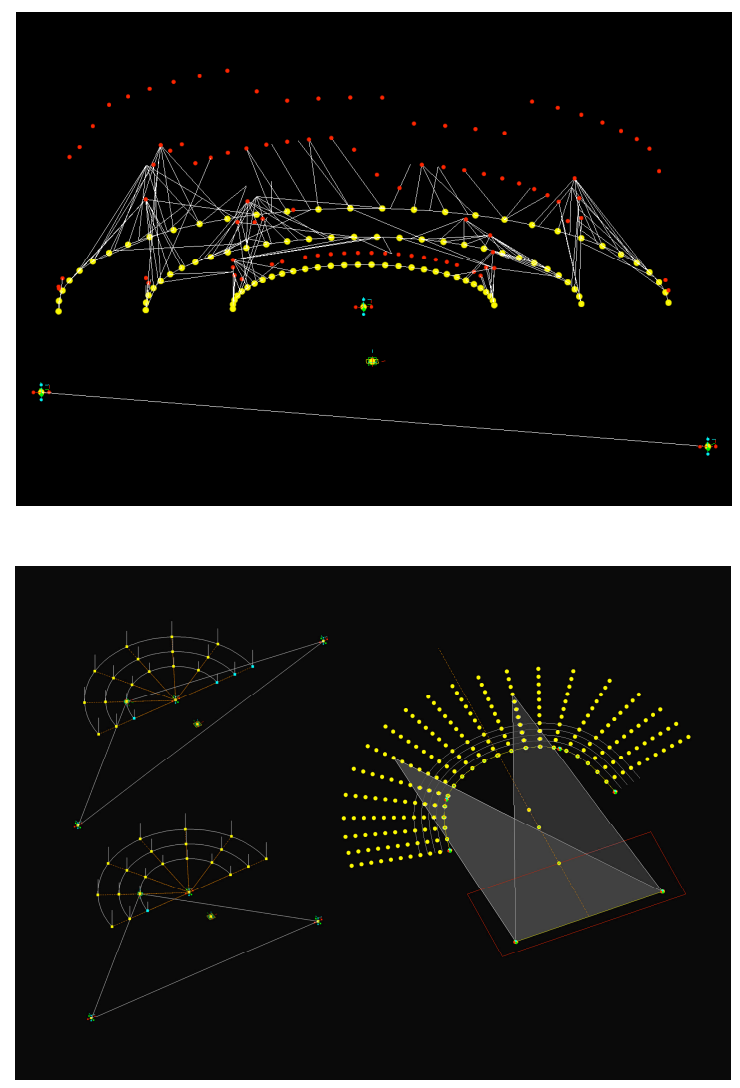

Figure 8: Views of a parametric model for theatre layout (Matthew Jogan) (with permission of author).

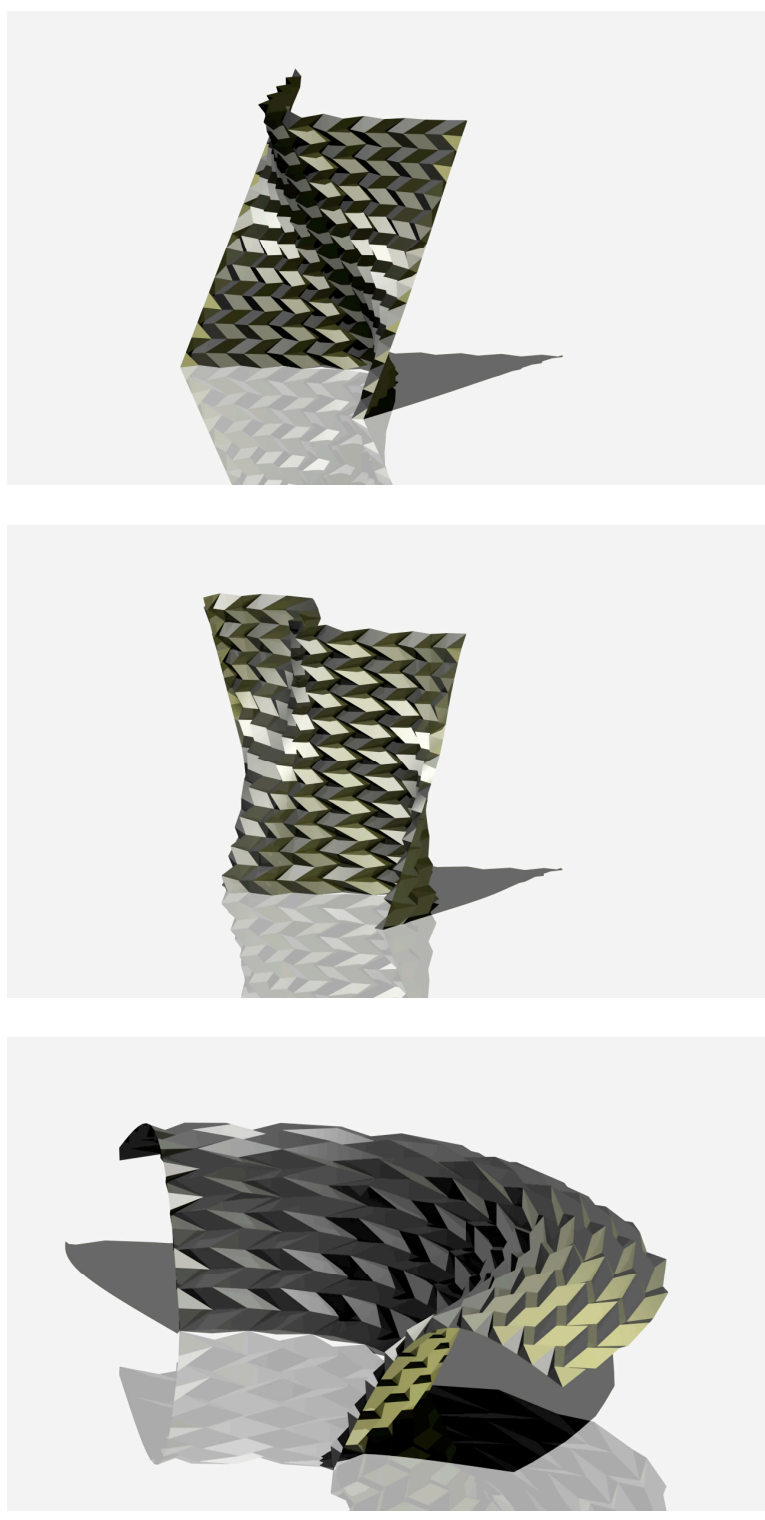

Figure 9: Views of a parametric model for the curved surface building skin. (Neal Panchuk) (with permission of author).

An example from the CDRN workshop is that of Neal Panchuk, who wrote:

The model developed here (Figure 9) consists of a variable chevron pattern based structural system that is based on the Miura-ori folding pattern found in nature and developed by Koryo Miura, a Japanese space scientist. It is intended that the chevron system be able to effectively populate and configure each individual chevron element to suit the local surface conditions. From this model I would like to generate a number of outputs. The first would be the efficient unfolding 
and flattening of the model into a fabrication model for laser cutting. The layout of the chevron units must occur in a sequential manner that I have not yet been able to create... The images provided represent the development of the chevron feature from the population and subsequent replication and offset of $U V$ points on a surface. Once the feature was derived it was possible to apply the chevron to any subsequent surface for construction.

\section{Parametric modelling across design and engineering}

The word "design" has two conflicting meanings. Design as the act of making proposals for change includes a wide variety of professional work, especially substantial aspects of engineering. Design is also used as a label for a large class of so-called "creative" disciplines in which the performance criteria are typically contextual, difficult to quantify and subject to continual debate. We use the word in both senses here in the contention that parametric modelling can be a substantial bridge between design and engineering. In the first sense (design as an act), parametric modelling can be used to model design criteria (at least to an approximation) into design representations, enabling exploration of wellperforming possibilities beyond that possible with conventional media. In the second sense (design as a disciplinary label), parametric modelling may well be a bridge that enables conversation between disciplines on the basis of shared goals and professional technique. Since engineers have long used parametric modelling, other designers clearly have much to learn from engineering experience. On the other hand, it may well be true that designers from fields other than engineering can contribute substantially to new uses of this important class of design software within engineering. At an educational level, many CDEN members have combined engineering and design students in their project courses. Parametric modelling may well be one device that makes such collaborations more interesting and effective. We believe that the converse is very likely to be true - that engineering expertise in parametric modelling can broaden avenues for students to learn to work across design disciplines.

\section{References}

[1] Revit. Accessed at www.autodesk.com/revit on 16 June 2006.

[2] CATIA. Accessed at www.3ds.com/productssolutions/brands/CATIA/ on 16 June 2006.

[3] Solid Works. Accessed at www.solidworks.com on 16 June 2006.

[4] R. Aish and R. Woodbury, "Multi-level Interaction in Parametric Design”, SmartGraphics, 5th International Symposium, SG2005, Lecture Notes in Computer Science 3638, Andreas Butz, Brian Fisher, Antonio Krüger and Patrick Oliver (eds). pp151-162. Springer. August 2005. 